\section{Fatores associados à recorrência da infecção do trato urinário em crianças}

\section{The factors associated to urinary tract infection recurrence in children}

\section{Abstract}

Objectives: to determine the frequency of recurrent urinary tract infection (UTI) and to evaluate the factors associated to UTI recurrence in children.

Methods: retrospective study of 95 patients with one year follow-up (68 girls and 27 boys, median of age three years old). The variables studied were: sex, age, fever, constipation, bacteria specimen, vesicoureteral reflux (VUR), abnormalities of renal Dimercaptosuccinic acid (DMSA) scintigraphy.

Results: Recurrent UTI occurred in $49.5 \%$ children (19 with normal urinary tract, 19 with VUR and 9 with stenosis of the ureteropelvic junction ). Comparing data of the group with recurrent UTI and the group without recurrent UTI the following was determined: there were no significant differences between sexes, presence of fever, constipation and abnormalities in renal DMSA scintigraphy. Recurrent UTI was significantly higher in symptomatic children $\leq 1$ year old, of those with $\leq 2$ years old, with VUR, with bacteria other than Escherichia coli and the ones not receiving antibacterial prophylaxis. Risk factors for UTI recurrence were age $\leq 2$ years old (Odds ratio $=3.83)$ and vesicoureteral reflux $-($ Odds ratio $=$ 4.95).

Conclusions: due to the high recurrent UTI frequency the regular follow-up of children with risk factor for recurrent UTI is essential.

Key words Child, Recurrent urinary tract infection, Risk factors
Márcia C. Riyuzo1

Célia S. Macedo2

Herculano D. Bastos3

1-3 Disciplina de Nefrologia Pediátrica. Departamento de Pediatria Faculdade de Medicina de Botucatu. Universidade Estadual Paulista. Distrito de Rubião Júnior s.n. Campus de Botucatu. Botucatu, SP, Brasil. CEP: 18.618-970. E-mail: mriyuzo@fmb.unesp.br

\section{Resumo}

Objetivos: verificar a freqüencia de infecção urinária recorrente (ITU) e avaliar os fatores associados à recorrência da ITU em crianças.

Métodos: estudo retrospectivo de 95 pacientes com seguimento de um ano (68 meninas e 27 meninos, mediana de idade três anos). As variáveis estudadas foram: sexo, idade, febre, constipação, tipo de bactéria, refluxo vésico-ureteral (RVU), anormalidades na cintilografia renal com ácido dimercaptosuccínico (DMSA).

Resultados: infecção urinária recorrente ocorreu em $49,5 \%$ crianças (19 com trato urinário normal, $19 \mathrm{com}$ $R V U$ e 9 com estenose da junção pielocalicial). Comparando o grupo com ITU recorrente com o grupo sem ITU recorrente não se encontrou diferença significativa entre sexos, presença de febre, constipação e anormalidades na cintilografia renal com DMSA. A ITU recorrente foi significativamente maior nas crianças com um ano ou menos, naquelas menores de dois anos com $R V U$, nas com bactéria diferente da Escherichia coli e sem profilaxia antibacteriana. Os fatores de risco significativos para a recorrência ITU foram idade $\leq 2$ anos $(O R=3,83)$ e refluxo vésico-ureteral $(O R=4,95)$.

Conclusões: por causa da elevada freqüência de ITU recorrente é importante o seguimento regular de grupo de crianças com fatores de risco para ITU recorrente. Palavras-chave Criança, Infecção urinária recorrente, Fatores de risco 


\section{Introdução}

A infecção urinária é evento freqüente na infância, incidindo em 3 a $5 \%$ de crianças do sexo feminino e em 1 a $2 \%$ do masculino. ${ }^{1,2}$ Com o advento de antibióticos e modernos meios diagnósticos a mortalidade por essa doença atualmente se aproxima de zero. ${ }^{2}$ No entanto, existem consequiências da infecção urinária a longo prazo como desenvolvimento de cicatrizes renais ocasionando hipertensão arterial, deterioração da função renal e complicações na gestação. ${ }^{2}$ A maioria das crianças com infecção urinária tem prognóstico excelente, porém há pequeno grupo de pacientes com riscos de sérias complicações. Isso é observado em 10 a $15 \%$ dos casos, ocorre mais freqüentemente após infecções recorrentes e a gravidade da lesão renal permanente se associa ao número de episódios de pielonefrite. ${ }^{2-4}$

A recorrência da infecção urinária é comum na infância, ocorrendo em $25 \%$ dos recém-nascidos, em $30 \%$ a $50 \%$ das crianças maiores e essa porcentagem aumenta para 60 a $75 \%$ depois da segunda e terceira infecções.2,4-7 Recorrência é definida como segundo episódio de infecção urinária, não importando se devido a recidiva (recrudescência de infecção urinária não curada) ou a reinfecção. 5,8-10 A maioria das recorrências deve-se a reinfecções 5,8,10 Para definir a reinfecção é necessário demonstrar nova espécie bacteriana ou outro sorotipo da mesma bactéria na urina 5,8,10 Duas ou mais culturas negativas entre duas infecções causadas por patógenos idênticos também indicam reinfecção. Infecção urinária recorrente ou infecção urinária de repetição é aquela na qual o paciente apresenta dois ou mais episódios infecciosos em seis meses 6,7 ou pelo menos três episódios em um ano. 8,10

Em vista da grande ocorrência de infecção urinária recorrente em crianças e a sua influência na formação de lesões renais, o objetivo deste estudo foi investigar, em crianças, a freqüência dessa condição e os fatores a ela associados.

\section{Métodos}

Estudo retrospectivo de 95 dentre 159 pacientes com infecção urinária, atendidos na disciplina de Nefrologia Pediátrica do Departamento de Pediatria da Faculdade de Medicina de Botucatu, da Universidade Estadual Paulista (UNESP),Botucatu, São Paulo, Brasil, no período entre janeiro de 1992 a dezembro de 2001. Foram atendidas 159 pacientes com infecção urinária, mas 64 foram excluídas do estudo - seis portadoras de mielomeningocele e seis com válvula de uretra posterior submetidas à correção cirúrgica prévia; duas com bexiga neurogênica; uma com síndrome de Prune Belly e 49 com investigação por imagem incompleta ou com seguimento inferior a um ano.

Para o diagnóstico da infecção urinária, após assepsia adequada, a urina foi coletada preferentemente por cateterização vesical ou jato intermediário em crianças menores de dois anos e por jato intermediário nas crianças maiores. ${ }^{11,12}$ No seguimento dos pacientes as culturas de urina foram realizadas a cada seis a oito semanas ou na presença de febre e/ou sintoma sugestivo de infecção urinária.

Após o tratamento da infecção urinária, para todos os pacientes foram prescritos medicamentos profiláticos (cefalexina nas crianças menores de três meses de idade; cefalexina ou nitrofurantoína ou ácido nalidíxico para as maiores de três meses) até a conclusão do estudo, por imagem. Em todos os pacientes a avaliação do trato urinário foi realizada pelo ultra-som renal ou pela urografia excretora e pela uretrocistografia miccional. A cintilografia renal com 99mTc ácido dimercaptosuccínico (DMSA), para diagnóstico de cicatriz renal, foi realizada em 74 pacientes $(77,9 \%)$. O tempo médio de obtenção completa dos resultados pelo estudo por imagem foi de $3,5 \pm 2,5$ meses.

Os pacientes foram divididos em dois grupos: os que apresentaram infecção urinária recorrente $(\mathrm{n}=$ 47) e os que não apresentaram infecção urinária recorrente $(n=48)$. O tempo de seguimento foi de um ano.

Os parâmetros avaliados foram: sexo; idade no início dos sintomas ( $\leq 1$ ou $>1$ ano); idade no início do seguimento ( $\leq 2$ ou $>2$ anos); presença de febre, constipação intestinal, refluxo vésico-ureteral e anormalidades na cintilografia renal com $99 \mathrm{mTc}$ DMSA e o agente etiológico. Na história clínica a febre foi caracterizada como temperatura axilar igual ou superior a $38,5^{\circ} \mathrm{C}$ e a constipação intestinal como a presença de alterações na freqüência, na consistência, no tamanho e/ou dificuldade na eliminação de fezes. Constipação também foi diagnosticada pela evidência de fezes retidas na ampola retal na radiografia simples do abdome do exame de uretrocistografia miccional. Definiu-se infecção urinária recorrente quando o paciente apresentou dois ou mais episódios infecciosos em seis meses.6,7

Os dados obtidos foram expressos como porcentagem, média desvio padrão ou mediana. Na análise dos dados utilizou-se o software SAS, versão 6.12 (SAS Institute, Cary, NC). Utilizou-se o teste Quiquadrado ou teste exato de Fisher, quando pertinente, para o estudo das associações entre as 
variáveis. Os dados que na análise univariada apresentaram valor de $p<0,25$ foram posteriormente submetidos à análise multivariada, utilizando o modelo de regressão logística, com a finalidade de avaliar simultaneamente a influência das diversas variáveis na recorrência da infecção urinária. ${ }^{13}$ A significância estatística foi estabelecida ao nível de 5\%.

O estudo foi aprovado pelo Comitê de Ética em Pesquisa do Hospital das Clínicas da Faculdade de Medicina de Botucatu, da UNESP.

\section{Resultados}

As características clínicas, o agente etiológico e o estudo por imagem das crianças com ou sem infecção urinária recorrente estão expressos nas Tabelas 1 , 2 e 3.

No período estudado, 47 de $95(49,5 \%)$ pacientes apresentaram infecção urinária recorrente.

Infecção urinária recorrente ocorreu em 63,8\% das meninas e em $36,2 \%$ dos meninos. A comparação entre crianças com e sem infecção urinária recorrente revelou semelhante proporção dos sexos feminino (30/47) $(63,8 \%)$ com infecção recorrente versus $(38 / 48)(79,2 \%)$ sem infecção recorrente, $p=0,95)$ e masculino (17/47) $(36,2 \%)$ com infecção recorrente versus $(10 / 48)(20,8 \%)$ sem infecção recorrente, $p=0,82$ ).

A idade no início dos sintomas variou entre 1 mês a 9 anos e 10 meses. Observou-se maior proporção de crianças com idade um ano no início dos sintomas que apresentaram infecção urinária recorrente $(p=0,007)$.

A idade dos pacientes no momento da consulta médica variou entre 1 mês a 11 anos e 3 meses. A recorrência da infecção urinária foi significativamente maior nos pacientes com idade $\leq 2$ anos no início do seguimento $(p=0,02)$.

Não se observou diferença estatística entre a proporção dos pacientes com ou sem infecção urinária recorrente que apresentaram febre $(p=0,19)$.

Não se observou diferença estatística entre a proporção dos pacientes com ou sem constipação intestinal que apresentaram infecção urinária recorrente $(p=0,08)$.

\section{Tabela 1}

\begin{tabular}{|c|c|c|c|c|c|c|}
\hline Característica & $\begin{array}{l}n \text { infecção } \\
\text { rrente (47) }\end{array}$ & $\begin{array}{l}\text { Sem infecção } \\
\text { recorrente (48) }\end{array}$ & $\begin{array}{l}\text { Qui-quadrado ou } \\
\text { teste exato Fisher (p) }\end{array}$ & $\begin{array}{c}\text { Intervalo de } \\
\text { de Confiaça } 95 \%\end{array}$ & Odds ratio & $p$ \\
\hline \multicolumn{7}{|l|}{ Sexo } \\
\hline Masculino & 17 & 10 & 0,82 & & & \\
\hline Feminino & 30 & 38 & 0,95 & & & \\
\hline Idade início dos sintomas & & & 0,007 & & & \\
\hline$\leq 1$ ano & 36 & 26 & & & & \\
\hline$>1$ ano & 11 & 21 & & & & \\
\hline Idade na consulta & & & 0,02 & $1,493-9,840$ & 3,833 & 0,005 \\
\hline$\leq 2$ anos & 27 & 19 & & & & \\
\hline$>2$ anos & 20 & 29 & & & & \\
\hline Presença de febre & 35 & 41 & 0,19 & & & \\
\hline Presença de constipação intestinal & 25 & 17 & 0,08 & & & \\
\hline Agente etiológico & & & 0,011 & & & \\
\hline Escherichia coli & 27 & 40 & & & & \\
\hline Klebsiella & 39 & 2 & & & & \\
\hline Proteus & 7 & 4 & & & & \\
\hline Enterobacter $s p$ & 3 & 2 & & & & \\
\hline Pseudomonas & 1 & 0 & & & & \\
\hline Trato urinário normal & 19 & 11 & & & & \\
\hline Refluxo vésico-ureteral & 19 & 35 & 0,03 & $1,902-12,927$ & 4,959 & 0,05 \\
\hline Estenose da junção ureteropiélica & 9 & 2 & & & & \\
\hline Presença de cicatriz renal/n* & $17 / 31$ & $22 / 43$ & 0,59 & & & \\
\hline
\end{tabular}

${ }^{*} \mathrm{n}=$ Números de exames realizados 
Tabela 2

Características clínicas, laboratoriais e do estudo por imagem de crianças sem infecção urinária recorrente.

\begin{tabular}{|c|c|c|c|c|c|}
\hline \multirow[t]{2}{*}{ Característica } & \multirow{2}{*}{$\begin{array}{l}\text { Trato urinário normal } \\
\qquad 11(22,9 \%)\end{array}$} & \multirow{2}{*}{$\begin{array}{l}\text { Refluxo vésico-ureteral } \\
\qquad 35(72,9 \%)\end{array}$} & \multirow{2}{*}{$\begin{array}{c}\text { Junção pielocalicial } \\
\text { não obstrutiva } 2(4,2 \%)\end{array}$} & \multicolumn{2}{|c|}{ Total } \\
\hline & & & & $\mathrm{n}$ & $\%$ \\
\hline \multicolumn{6}{|l|}{ Sexo } \\
\hline Masculino & 1 & 8 & 1 & 10 & 20,8 \\
\hline \multicolumn{6}{|c|}{ Idade início dos sintomas } \\
\hline$\leq 1$ ano & 6 & 19 & 1 & 26 & 54,2 \\
\hline$>1$ ano & 5 & 16 & 1 & 22 & 45,8 \\
\hline \multicolumn{6}{|l|}{ Idade na consulta } \\
\hline$\leq 2$ anos & 4 & 15 & 0 & 19 & 39,6 \\
\hline$>2$ anos & 7 & 20 & 2 & 29 & 60,4 \\
\hline Presente & 10 & 29 & 2 & 41 & 85,4 \\
\hline Ausente & 1 & 6 & 0 & 7 & 14,6 \\
\hline \multicolumn{6}{|c|}{ Constipação intestinal } \\
\hline Presente & 10 & 7 & 0 & 17 & 35,4 \\
\hline Ausente & 1 & 28 & 2 & 31 & 64,6 \\
\hline \multicolumn{6}{|l|}{ Cicatriz renal* } \\
\hline Presente & 4 & 17 & 1 & 22 & 51,2 \\
\hline Ausente & 2 & 18 & 1 & 21 & 48,8 \\
\hline \multicolumn{6}{|l|}{ Agente etiológico } \\
\hline Escherichia coli & 10 & 28 & 2 & 40 & 83,3 \\
\hline Proteus sp & 1 & 3 & 0 & 4 & 8,4 \\
\hline Enterobacter sp & 0 & 2 & 0 & 2 & 4,2 \\
\hline Klebsiella sp & 0 & 2 & 0 & 2 & 4,2 \\
\hline
\end{tabular}

* Número de exames realizados $=43$

Infecção urinária recorrente foi observada em 9 de 11 crianças com estenose da junção ureteropiélica, em 19 de 30 crianças com trato urinário normal e em 19 de 54 crianças com refluxo vésicoureteral. Houve maior proporção de pacientes com refluxo vésico-ureteral que apresentaram infecção urinária recorrente $(p=0,03)$ comparada aos pacientes sem refluxo vésico-ureteral. Infecção urinária recorrente foi observada em 38,7\% (12/31) pacientes com refluxo unilateral e em $33,3 \%(7 / 21)$ com refluxo bilateral; em $23,8 \%(5 / 21)$ com refluxo de grau I ou II, em 33,3\% (8/21) de grau III e em $66,7 \%(6 / 9)$ de grau IV ou V. Nos pacientes com infecção urinária recorrente não houve diferença estatística significativa entre os portadores de refluxo unilateral ou bilateral $(p=0,52)$, mas houve maior proporção de pacientes com refluxo de grau IV ou V $(p=0,04)$.

Não se observou diferença estatísticamente significante entre a proporção dos pacientes com infecção urinária recorrente que apresentaram exame alterado de cintilografia renal com DMSA $(p=0,59)$.

Observou-se maior proporção de agente etiológico diferente da Escherichia coli nas crianças com infecção urinária recorrente comparada às sem infecção recorrente (agente etiológico Escherichia coli $=27$ e outro $=20$ nas crianças com infecção urinária recorrente versus agente etiológico Escherichia coli $=40$ e outro $=8$ nas crianças sem infecção urinária recorrente, $p=0,011$ ).

Nos 47 pacientes com infecção urinária recorrente ocorreram 95 episódios infecciosos. Escherichia coli foi o agente etiológico em 57,9\% dos episódios (55/95), Klebsiella em 16,8\% (16/95), Proteus sp em 12,6\% (12/95), Pseudomonas sp em 7,3\% (7/95), Citrobacter sp em 3,1\% (3/95), Enterococos em 1,0\% (1/95) e Streptococcus viridans em 1,0\% (1/95). Os meninos apresentaram 44 episódios infecciosos e as meninas 51 episódios. Nos meninos Escherichia coli foi o patógeno em 18 


\begin{tabular}{|c|c|c|c|c|}
\hline \multirow[t]{3}{*}{ Parâmetros } & \multicolumn{4}{|c|}{ Análise estatística } \\
\hline & \multirow{2}{*}{$\begin{array}{l}\text { Qui-quadrado ou } \\
\text { teste Exato de Fisher }(p)\end{array}$} & \multicolumn{3}{|c|}{ Análise multivariada } \\
\hline & & IC95\% & Odds ratio & $p$ \\
\hline Sexo feminino & 0,95 & & & \\
\hline Sexo masculino & 0,82 & & & \\
\hline Idade início dos sintomas $\leq 1$ ano $/>1$ ano & 0,007 & & & \\
\hline Idade no seguimento $\leq 2$ anos $/>2$ anos & 0,02 & $1,493-9,840$ & 3,833 & 0,0052 \\
\hline Presença de febre & 0,19 & & & \\
\hline Presença de constipação intestinal & 0,08 & & & \\
\hline Presença de refluxo vésico ureteral & 0,03 & $1,902-12,927$ & 4,959 & 0,05 \\
\hline Presença de cicatriz renal & 0,59 & & & \\
\hline Bactéria diferente Escherichia coli & 0,011 & & & \\
\hline
\end{tabular}

episódios, Proteus sp em 12, Klebsiella em 12 e Citrobacter $s p$ em 2. Nas meninas Escherichia coli foi o patógeno em 37 episódios, Klebsiella em 4, Pseudomonas sp em 7, Enterococos em 1, Streptococcus viridans em 1 e Citrobacter sp em 1.

Após o estudo por imagem do trato urinário os medicamentos profiláticos foram mantidos em 84 pacientes (88,4\%): 54 com refluxo vésico-ureteral, 8 com estenose da junção uretero-piélica e 22 com trato urinário normal. Infecção urinária ocorreu em $44,0 \%$ (37/84) dos pacientes recebendo medicamento profilático (em 19 com refluxo vésicoureteral, 6 com estenose da junção uretero- piélica e 12 com trato urinário normal) e em 90,9\% (10/11) sem medicamento profilático ( 7 com trato urinário normal e 3 com estenose da junção uretero-piélica). Houve maior e significativa proporção de crianças sem medicamento profilático que apresentou infecção urinária recorrente (37 crianças com profilático e 10 sem profilático que apresentaram infecção urinária recorrente versus 47 crianças com profilático e 1 sem profilático que não apresentaram infecção urinária recorrente, $p=0,009)$.

Os resultados da análise multivariada revelaram que os fatores de risco significativos na recorrência da infecção urinária foram a idade $\leq 2$ anos no início do seguimento no Serviço (intervalo $95 \%$ de confiança, 1,493-9,840, Odds ratio $=3,833, p=0,005) \mathrm{e}$ a presença de refluxo vésico-ureteral (intervalo $95 \%$ de confiança, 1,902-12,927, Odds ratio $=4,959, p=$ $0,05)$. Crianças com a idade $\leq 2$ anos no início do seguimento no Serviço apresentam risco de 3,83 vezes maior que as com idade $>2$ anos; e crianças com refluxo vésico-ureteral apresentam risco de 4,95 vezes maior que aquelas sem refluxo vésico-ureteral para apresentarem infecção urinária recorrente (Tabela 3).

\section{Discussão}

Infecção urinária recorrente é descrita em 12 a 75\% dos pacientes. 1,2,4,5,7,14-16 A freqüência observada em nosso estudo foi semelhante a descrita na literatura.

As meninas têm maior probabilidade de apresentar infecção urinária recorrente que os meninos. 2,4,5 A recorrência de infecção urinária após a primo-infecção é descrita em $50 \%$ das meninas durante o primeiro ano e em $75 \%$ dos casos durante dois anos de seguimento.5,8 Não há dados comparativos para o sexo masculino. $2,8,17$

Nos meninos foi observado alta porcentagem de recorrência de infecção urinária em cerca de $60 \%$ dos casos na faixa etária $\leq 2$ anos. A presença do prepúcio íntegro pode ter influenciado o resultado. A infecção urinária ocorreu em $86 \%$ dos meninos não circuncidados no primeiro ano de vida. 18 É descrita maior freqüência de infecção urinária em meninos não circuncidados e estima-se que a presença de prepúcio íntegro eleva o risco de infecção urinária entre 3,7 e 11 vezes. 5,7,17,19 Semelhante ao descrito na literatura, foi observado proporção semelhante de infecção urinária recorrente entre os sexos masculino e feminino. 7,14

Em crianças com infecção urinária no primeiro 
ano de vida, a idade abaixo de seis meses na primeira infecção foi significativamente associada com infecção recorrente e foi fator de risco para recorrência.7,14 No presente estudo verificou-se que idade da criança inferior a dois anos no início do seguimento esteve associada à recorrência da infecção urinária e foi fator de risco para recorrência, resultados semelhantes aos descritos na literatura.

A presença de febre não esteve associada à recorrência de infecção urinária, fato relatado na literatura. 14

Há associação entre constipação intestinal e infecção urinária recorrente. ${ }^{2,5,17,20-22}$ Essa associação é descrita em $11 \%$ de pacientes, principalmente em meninas. ${ }^{22}$ Com o tratamento da constipação ocorreu o desaparecimento de infecção urinária em $100 \%$ dos casos sem anormalidades urológicas. 22 Não foi observado associação significativa entre constipação intestinal e infecção urinária recorrente. Esse fato pode ser decorrente da presença de maior proporção de pacientes com idade $\leq 2$ anos e/ou com refluxo vésico-ureteral nos pacientes sem constipação intestinal.

A prevalência de refluxo vésico-ureteral entre crianças com infecção urinária recorrente varia entre $20 \%$ a $50 \% .2,5,14,23$ Os dados obtidos no presente estudo são semelhantes aos descritos na literatura. $\mathrm{O}$ papel do refluxo vésico-ureteral em predispor à infecção recorrente é controverso. $2,14,24$ Os graus III a $\mathrm{V}$ de refluxo e refluxo bilateral são fatores associados à recorrência da infecção urinária.2,14,16,17 No presente estudo a presença de refluxo vésico-ureteral foi fator de risco para infecção urinária recorrente. Refluxo bilateral não se associou à infecção urinária recorrente enquanto os refluxos de grau IV e V contribuíram para a recorrência da infecção.

Em crianças menores de cinco anos, anormalidades na cintilografia renal com DMSA na primeira infecção urinária é um dos fatores de risco para infecção urinária recorrente. ${ }^{14}$ Meninas com cicatrizes renais continuam a apresentar alta proporção de recorrências de pielonefrite após 10 anos de idade. 25 No presente estudo esse fato não foi constatado.

A bactéria mais freqüentemente responsável pela infecção urinária é Escherichia coli com $80 \%$ dos casos. 2,5,17 Escherichia coli foi identificada em $67 \%$ dos pacientes com infecção urinária recorrente. ${ }^{14}$ Em crianças com infecção urinária recorrente e naquelas tratadas com antibióticos profiláticos há maior incidência de infecções urinárias causadas por Proteus sp, Klebsiella sp e Enterobacter $s p . .^{5}$ Os dados do presente estudo são semelhantes aos descritos na literatura.

A taxa de novas infecções urinárias é influenciada pelo uso e duração de profilaxia antibacteriana com dose baixa para prevenir recorrências em pacientes de alto risco. ${ }^{2}$ A quimioprofilaxia é eficaz na prevenção de infecção urinária, no entanto, devese considerar remota a prescrição da quimioprofilaxia em crianças com infecção recorrente e trato urinário normal.16,17 Presença de refluxo vésicoureteral, má formações urinárias obstrutivas, distúrbios funcionais da bexiga e resíduo urinário miccional são fatores importantes na recorrência da infecção urinária.2,17 Pode-se indicar a quimioprofilaxia nos casos de infecção urinária recorrente associada a condições que predispõem à estase urinária como na constipação intestinal e disfunção miccional, enquanto é realizado o tratamento dessas condições; igualmente no refluxo vésico-ureteral.2,17

Em conclusão, com os dados obtidos no presente estudo, foi observada elevada frequiência de infecção urinária recorrente, principalmente em crianças cujos sintomas se iniciam na idade abaixo de um ano, nas menores de dois anos de idade, nas portadoras de refluxo vésico-ureteral, nas que apresentam o agente etiológico diferente da Escherichia coli e naquelas que não utilizavam profilaxia antibacteriana. É sugerido o seguimento regular de crianças com infecção urinária, com controles de cultura de urina a cada seis ou oito semanas, principalmente em menores de dois anos de idade e/ou portadores de refluxo vésico-ureteral para o tratamento da recorrência de infecção urinária.

\section{Agradecimentos}

À professora Assistente Doutor Liciana Vaz de Arruda Silveira do Departamento de Bioestatística do Instituo de Biociências da UNESP, Botucatu.

\section{Referências}

1. Stull LT, Lipuma JJ. Epidemiology and natural history of urinary tract infection in children. Med Clin North Am. 1991; 75: 287-97.
2. Hansson S, Jodal U. Urinary tract infection. In: Avner ED, Harmon WE, Niaudet P, editors. Pediatric nephrology. 5th ed. Philadelphia: Lippincott Williams \& Wilkins; 2004. p. 1007-25 
3. Jodal U. The natural history of bacteriuria in childhood. Infect Dis Clin North Am. 1987; 1: 713-29.

4. Clarke SE, Smellie JM, Gurney S, West DJ. Technetium99m-DMSA studies in pediatric urinary infection. J Nucl Med. 1996; 37: 823-8.

5. Hellerstein S. Recurrent urinary tract infections in children. Pediatr Infect Dis. 1982; 1: 271-81.

6. Feld LG, Greenfield SP, Ogra PL. Urinary tract infections in infants and children. Pediatr Rev. 1989; 11: 71-7.

7. Mangiarotti P, Pizzini C, Fanos V. Antibiotic profilaxis in children with relapsing urinary tract infections: review. J Chemother. 2000; 12: 115-23.

8. Shapiro E, Elder JS. The office management of recurrent urinary tract infection and vesicoureteral reflux in children. Urol Clin North Am. 1998; 25: 725-34.

9. Lacombe J. Clinical evidence: urinary tract infection in children. BMJ. 1999; 319: 1173-5.

10. Riccabona M. Management of recurrent urinary tract infection and vesicoureteral reflux in children. Curr Opin Urol. 2000; 10: 25-8.

11. American Academy of Pediatrics. Practice parameter: the diagnosis, treatment, and evaluation of the initial urinary tract infection in febrile infants and young children. Pediatrics. 1999; 103: 843-51.

12. Macedo CS, Riyuzo MC, Fekete SMW, Trindade AAT, Bastos HD. Infecção do trato urinário na criança-estudo retrospectivo. Rev Paul Pediatr. 2002; 20: 169-78.

13. Collett D. Modeling binary data. London: Chapman and Hall; 1991.

14. Panaretto KS, Craig JC, Knight JF, Howman-Giles R, Sureshkumar P, Roy LP. Risk factors for recurrent urinary tract infection in preschool children. J Paediatr Child Health. 1999; 35: 454-9.
15. Winberg J, Bergstrom T, Jacobsson B. Morbidity, age and sex distribution, recurrences and renal damage in symptomatic infection in childhood. Kidney Int. 1975; 8 (Suppl): S101-6.

16. Nuutinen M, Uhari M. Recurrence and follow-up after urinary tract infection under the age of one year. Pediatr Nephrol. 2001; 16: 69-72.

17. Koch VH, Zucolotto SMC. Infecção do trato urinário. Em busca de evidências. J Pediatr (Rio J) 2003; 79 (Supl 1): S97-106.

18. Schoen EJ, Colby CJ, Ray GT. Newborn circumcision decreases incidence and costs of urinary tract infections during the first year of life. Pediatrics. 2000; 105: 789-93.

19. Nayer A. Circumcision for the prevention of significant bacteriuria in boys. Pediatr Nephrol. 2001; 16: 1129-34.

20. Neumann PZ, deDomenico IJ, Nogrady MB. Constipation and urinary tract infection. Pediatrics. 1973; 52: 241-5.

21. O'Reagan S, Yasbeck S, Schick E. Constipation, bladder instability, urinary tract infection syndrome. Clin Nephrol. 1985; 23: 152-4.

22. Loening-Baucke V. Urinary incontinence and urinary tract infection and their resolution with treatment of chronic constipation of childhood. Pediatrics. 1997; 100: 228-32.

23. Le Saux N, Pham B, Moher D. Evaluating the benefits of antimicrobial prophylaxis to prevent urinary tact infections in children: a systematic review. Can Med Assoc J. 2000; 163: 523-9.

24. Garin EH, Orta-Sibu N, Campos A. Primary vesicoureteral reflux in childhood. Adv Pediatr. 2002; 49: 341-57.

25. Martinell J, Claesson I, Lindin-Janson G, Jodal U. Urinary infection, reflux and renal scarring in females continuously followed for 134-38 years. Pediatr Nephrol. 1997; 11: 10820 .

Recebido em 10 de outubro de 2006

Versão final apresentada em 16 de fevereiro de 2007

Aprovado em 4 de abril de 2007 